\title{
COVID-19: pathogenic mechanism, detection, and treatment
}

\author{
Xiaoya Bai ${ }^{1, \mathrm{a}, \dagger}$, Chenghao $\mathrm{Li}^{2, \mathrm{~b}, \dagger}$, Yueyan $\mathrm{Li}^{3, \mathrm{c}, \dagger}$, Yujian $\operatorname{Lin}^{4, \mathrm{~d}, \uparrow}$ \\ ${ }^{1} \mathrm{Xi}$ 'an Jiaotong-Liverpool University, Faculty of science \\ ${ }^{2}$ North Carolina State University, Microbiology: Microbial Health Science \\ ${ }^{3}$ Peking University, Health Science Center \\ ${ }^{4}$ University of British Columbia, Faculty of land and food systems \\ ${ }^{\dagger}$ These authors contributed equally.
}

\begin{abstract}
COVID-19, which broke out in 2019 in China, is caused by SARS-CoV-2. According to the latest WHO real-time statistics, as of 1:19 p.m. BST on April 22, there were 142557268 cumulative new confirmed cases of coronavirus pneumonia and 3033798 cumulative deaths worldwide. Therefore, it is significant to understand the structure and the pathogenic mechanism of SARS-CoV-2, which is helpful to understand the diagnosis and treatment of sars-cov-2. We primarily investigate related sources on Pubmed and related scholar websites. We found some antiviral drugs such as lopinavir, chloroquine, and its derivative hydroxychloroquine, remdesivir, and arbidol have great potential in the treatment of COVID-19. However, some of them have a serious adverse reaction, reminding us to use them with caution.
\end{abstract}

\section{Introduction}

COVID-19 is a type of pneumonia caused by coronavirus infection. The epidemic started in late 2019, and the situation has not slowed down until now. According to the latest real-time statistics from WHO, as of 1:19 p.m. BST on April 22, there were a cumulative total of 14,257,268 new confirmed cases of coronavirus pneumonia and a cumulative total of 303,798 deaths worldwide. The special structure of coronavirus is the main reason for the pathogenic mechanism. Coronaviruses dump multiple sites in the patient's body, including the lungs, cardiovascular, kidneys, and intestines. Three proteins are wrapped around the periphery of the new coronavirus; they are the spike glycoprotein, the envelope protein, and the matrix glycoprotein. Even though the genes of the neocoronavirus are known, it is constantly mutating to adapt to new environments, which poses many difficulties for scientists to study. To cope with the ever-mutating neocoronavirus, scientists have explored several detection methods. Diagnostic methods for neo-coronaviruses include antibody assays such as enzyme-linked immunosorbent assay (ELISA), colloidal gold immunochromatographic assay, two lateral flow immunoassays, and nucleic acid assays such as reverse transcription-PCR, RT-PCR, and neo-coronavirus. RTPCR), transcription-mediated amplification (TMA), clustered regularly interspaced short text repeats (CRISPR), computed tomography (CT), antisense oligonucleotide-directed electrochemical biosensor wise. Current treatments for coronaviruses include antiviral drugs and vaccines. The drugs are lopinavir, chloroquine $\&$ hydroxychloroquine, remdesivir, arbidol. Vaccines are developed in three ways, i.e., genetic, subunit, and whole microorganism. This article reviews the progress of research on COVID-19 treatment and vaccination.

\section{COVID-19 structure}

The virus was observed under the electron microscope using a negative stain. The viruses are generally spherical. Some are polymorphic and have a diameter between 75-160 $\mathrm{nm}$. Viral particles have morphological protrusions similar to a corona at the edge, about 9-12 nm, and look like a crown. Extracellular free virus particles and cytoplasmic membrane vesicles filled with virus particles were found in ultrathin sections of the human airway epithelium. This morphology observed is consistent with the coronaviridae.

\subsection{The three outmost proteins}

There are three proteins at the outmost of the COVID-19, which are spike glycoprotein, envelope protein, and matrix glycoprotein [ 1 ]. Spike glycoprotein is responsible for receptor binding and membrane fusion and is targeted by host neutralizing antibodies.

The $\mathrm{S}$ protein is $180-200 \mathrm{kDa}$ in size and consists of an extracellular N-terminus, a transmembrane (TM) domain anchored to the viral membrane, and a short intracellular C-terminal segment. the $\mathrm{S}$ protein trimer visually forms the characteristic spherical, crown-like halo around the viral particle [2]. In the native state, the 
$\mathrm{CoV} \mathrm{S}$ protein exists as an inactive precursor. Once the virus interacts with the host cell, the $\mathrm{S}$ protein undergoes extensive structural rearrangement. The target cell protease activates the $\mathrm{S}$ protein by cleaving it into $\mathrm{S} 1$ and S2 subunits, altering the metastable pre-fusion conformation and allowing the virus to fuse with the host cell membrane. This is necessary for the activation of the membrane fusion domain upon viral entry into the target cell. A large number of glycosylated $\mathrm{S}$ proteins cover the surface of SARS-CoV-2 and bind to the host cell receptor angiotensin-converting enzyme 2 (ACE2), mediating viral cell entry. When the $\mathrm{S}$ protein binds to the receptor, the type 2 TMProtease serine 2 (TMPRSS2) located on the host cell membrane facilitates viral entry by activating the $\mathrm{S}$ protein. The virus enters the cell at first. Then, it releases viral RNA, translates the polyprotein, replicates and transcriptionally generates the viral RNA genome. And then it synthesizes, assembles, and packages structural proteins in the host cell. Finally, the viral particles are released. Similar to other coronaviruses, the $\mathrm{S}$ protein of SARS-CoV-2 is cleaved into $\mathrm{S} 1$ and $\mathrm{S} 2$ subunits by a cellular protease, and the serine protease TMPRSS2 is used as a protein primer. Although the cleavage site of SARS-CoV is known, the cleavage site of SARS-CoV-2 S has not been reported.

Envelope protein is important for virus infectivity. The $\mathrm{CoV}$ envelope (E) protein is a small, integral membrane protein involved in several aspects of the viral life cycle, such as assembly, budding, envelope formation, and pathogenesis. CoVs lacking $\mathrm{E}$ are therefore promising vaccine candidates [3]. The high mortality rate of $\mathrm{CoVs}$ and their ease of transmission suggest the need for more research on $\mathrm{CoV}$ molecular biology, which will contribute to the production of effective anti-coronaviral drugs for human CoVs and enzootic CoVs. Matrix glycoprotein is the most abundant structural protein and interacts with $\mathrm{E}$ to form.

\subsection{Kernel}

Helical nucleocapsid protein and genome are in the kernel. Helical nucleocapsid protein interacts with various proteins in viral or host cells and affects multiple pathways in host cells. It is an important multifunctional protein involved in processes such as viral infection, replication, and viral packaging.

The coronavirus nucleocapsid protein is a protein that encapsulates viral genomic RNA and forms part of the viral structure. The nucleocapsid protein of SARS-CoV is highly antigenic and has been associated with various host cell interactions. RNA has been shown to bind to the N-terminal domain (NTD), although recently, the Cterminal half of the protein has also been implicated in RNA binding [4]. To determine the molecular basis of this activity, we also solved the crystal structure of the NP248-365 region. Residues 248-280 form a positively charged groove similar to that in the nucleocapsid protein of infectious bronchitis virus (IBV). In addition, the positively charged surface area is larger in the SARS-CoV construct than in IBV. The interaction between residues $248-280$ and the rest of the molecule also stabilizes the formation of octamers in the asymmetric unit. The packing of octamers in the crystal forms two parallel basic helical grooves, which may be oligonucleotide attachment sites, and suggest a mechanism for helical RNA packing in the virus.

The genome of SARS-CoV-2 is a positive singlestranded RNA(+ssRNA) and approximately $30 \mathrm{~kb}$ in length. It has no error correction ability of DNA transcription and is prone to mutation.

\section{Pathogenic mechanism}

\subsection{Invasion}

Novel coronaviruses use highly glycosylated homologous trimeric $\mathrm{S}$ proteins to enter the host cell. The $\mathrm{S}$ proteins undergo various structural rearrangements before fusing the virus into the host cell's cell membrane. This process involves binding the S1 subunit of the virus to the host cell receptor, triggering the onset of trimer instability, which causes the S1 subunit to shed the S2 subunit to form a highly stable post-fusion structure.

\subsection{Hibernation}

SARS-CoV-2 then lies dormant in the nasal cavity, waiting for the right time to enter human cells. The key for the virus to select the nasal cavity is ACE2, which uses ACE2 as the receptor to infect cells, and ACE2, as an important member of the renin-angiotensin system (RAS), is widely distributed in all tissues of the body and plays an important role in maintaining the physiological functions of the cardiovascular system, the inflammatory response of the body, and lung injury. Therefore, the virus can enter the human cells by binding to ACE2.

\subsection{Effects on the human body}

\subsection{1 lung}

If the body's immune system fails to fight the new coronavirus, the virus travels up the trachea. It enters the lungs, where the bronchi repeatedly branch into many fine bronchioles, the ends of which expand into sacs surrounded by many small, prominent alveoli called alveoli [5]. In addition, the alveoli are rich in ACE2 receptors, so the new crown becomes severe, even fatal.

\subsubsection{Heart, blood vessels}

In a cohort study including 100 recently recovered patients with COVID-19 identified from a COVID-19 testing center, cardiac magnetic resonance imaging showed 78 patients $(78 \%)$ had cardiac involvement, and 60 patients $(60 \%)$ had persistent myocardial inflammation, according to a study in JAMA Cardiology [6]. This was independent of preexisting medical 
conditions, the severity and overall duration of acute illness, and time from initial diagnosis.

Feldstein and his team showed that in this series of 539 patients with MIS-C and 577 patients with severe COVID-19, the MIS-C patients were more likely to be 6 to 12 years old than the severe COVID-19 patients, to be non-Hispanic blacks, to have severe cardiovascular or skin mucosal involvement and to have more severe inflammation [7]. This means that the findings suggest a pattern of clinical presentation and organ involvement that distinguishes patients with MIS-C from those with severe acute COVID-19.

These findings suggest the need for scientists to continuously investigate the long-term cardiovascular consequences of COVID-19. In addition, infections can cause vasoconstriction, and patients experience localized ischemia - reduced blood flow to the extremities leading to swelling, pain, and tissue necrosis in the fingers. Vasoconstriction in the lungs also seems to explain a puzzling phenomenon in COVID-19 pneumonia: patients have extremely low blood oxygen levels but no respiratory distress. Perhaps at some point in the disease, the virus altered the delicate balance of hormones that help regulate blood pressure and constrict blood vessels entering the lungs. Thus, the constricted blood vessels, rather than the blocked alveoli, impede oxygen intake.

If COVID-19 targets blood vessels, this could explain why patients with cardiovascular disease (including diabetes and hypertension) are at higher risk of developing it. However, the mechanism behind the damage is unclear. Perhaps, similar to the nasal cavity and alveoli, the heart and blood vessels are rich in ACE2 receptors. Perhaps the lung confusion causes hypoxia to damage the blood vessels, or perhaps the cytokine storm destroys the heart like it does other organs.

\subsubsection{Brain}

In studies on inpatients with toxic metabolic encephalopathy, toxic metabolic encephalopathy (TME) was reported in 7-31\% of COVID-19 inpatients in 2019. TME occurs in one in eight COVID-19 inpatients and is typically a multifactorial disease, hypoxemia, sepsis, and uremia as the most common causes [8]. After correction for confounders, TME was associated with a $24 \%$ risk of in-hospital mortality.

\subsubsection{Intestine}

However, COVID-19 patients also present with GI symptoms and are often described in outpatients with milder disease. This study sought to characterize the clinical features of GI symptoms and mild disease severity in patients with COVID-19. Positive SARSCoV-2 viral RNA in stool samples and signs of colonic damage on endoscopy in patients with COVID-19 suggest that this coronavirus infects the GI tract.

The clinical features of GI symptoms and mild disease in COVID-19 patients were mild disease and one or more GI symptoms (diarrhea, nausea, and vomiting).
There were 206 patients with low-severity COVID-19, including 48 with GI symptoms alone, 69 with both GI and respiratory symptoms, and 89 with respiratory symptoms alone. Among the two groups with GI symptoms, 67 cases had diarrhea, and $19.4 \%$ of them had diarrhea as the first symptom during the course of the disease. Diarrhea lasted from 1 to $14 \mathrm{~d}$, with a mean duration of $5.4 \pm 3.1 \mathrm{~d}$ and the number of bowel movements $4.3 \pm 2.2$ times/d. $62.4 \%$ of the patients with gastrointestinal symptoms were complicated by fever. Patients with gastrointestinal symptoms were seen later than those with respiratory symptoms. However, patients with digestive symptoms had a longer duration from symptom onset to viral clearance and a higher rate of fecal virus positivity than patients with respiratory symptoms. A distinct subgroup of COVID-19 patients was more likely to have positive viral RNA tests in stool, longer delays before viral clearance. It delayed diagnosis compared to patients with respiratory symptoms only.

\section{Virus detection}

\subsection{Nucleus acid test}

\subsubsection{RT-PCR}

COVID-19, a new type of coronavirus, infects cells by attaching to ACE2 receptor cells in the cell surface and endocytosing them into cells. There are two common methods for detecting COVID-19: Nucleic Acid Tests and Antibody Tests. Nucleic acid tests amplify DNA, RNA or proteins of specific pathogen to detect specific pathogens. An Antibody Test detects antibodies produced by the immune system in response to infection to determine whether a person has ever had an illness or is asymptomatic.

The most common Nucleic Acid Amplification Tests today are RT-PCR. Common PCR promotes the division of DNA by using DNA polymerase and detects DNA by detecting primers and probes that bind to DNA polymerase. However, the usual PCR method cannot detect RNA viruses. On the other hand, RT-PCR utilizes RNA reverse transcriptase to reverse transcription. The RNA will be transcribed to DNA and then amplifies by standard PCR methods to detect RNA viruses [9]. In the experiment that use RT-PCR to detect COVID-19, the researchers mixed RNA with Taq polymerase, a reverse transcriptase/Taq mixture. By looking at the primers and probes mixed with RNA polymerase, the researcher determines whether the virus is COVID-19 and determines whether RT-PCR is suitable for academic and public laboratories. The results of RT-PCR assays are relatively reliable and are now one of the most common methods for detecting COVID-19.

\subsubsection{Transcription-mediated amplification (TMA)}

In addition to RT-PCR, scientists have discovered that Transcription-mediated amplification (TMA), a method that amplifies the number of target genes to detect 
COVID-19. TMA is a method that amplifies RNA by using RNA polymerase and reverse transcriptase, which is more rapid than PCR. Although the RT-PCR assay has a high sensitivity, the pathogenicity of COVID-19 still affected the result. At that time, TMA can help detect samples with uncertain results. TMA will add the VTM to the hology lysis tube solution and use the hologic Panther to analyze positive, negative, or invalid sample results [10]. In general, TMA is a highly sensitive method, which can help to detect uncertain RT-PCR results.

\subsubsection{CRISPR}

CRISPR is a recently emerged method that has the potential to replace RT-PCR assays. CRISPR is common biotechnology in gene editing, but it can also be used for COVID-19 nucleic acid detection. Activated Cas13a is used for reprogramming of CRISPR RNA in detection. Once the RNA target is identified, it cleaves nearby nontarget RNA and causes the death or degradation of nontarget cells near the target cell in the presence of the specific RNA. Scientists can specifically and rapidly detect targets by observing the breakdown of reporter RNA by cas13a-mediated collateral [11].

\subsubsection{CT}

The RT-PCR requires specialized equipment and professional workers, which increases the difficulty of a detection. However, CT is an effective method that saves time and convenience. According to the description of Trial Version 7: Diagnosis and Treatment Protocol for Novel Coronavirus Pneumonia from the Chinese Health Care Commission, chest images of patients with COVID-19 showed many small patchy shadows, after few days, both lungs will have glass opacities and infiltration [12]. By observing the chest image, it is easy and effective for the early detection of suspicious cases.

\subsubsection{Antisense Oligonucleotides Directed Electrochemical Biosensor Chip}

The Antisense Oligonucleotides Directed Electrochemical Biosensor Chip is biosensor that develop a rapid and low-cost COVID-19 detection method. This biosensor detects viral nucleocapsid phosphoprotein using gold nanoparticles. The sensor has a probe that reads the results of the nucleic acid test. Through experiments, the researchers demonstrated that in the presence of COVID-19, significant changes in the signal within the sensor could be observed in less than 5 minutes. For healthy populations, or those infected with other coronaviruses, the signal change is not significant, so this method has a high specificity, accuracy, and sensitivity [13].

\subsection{Antibody test}

\subsubsection{ELISA}

Besides the nucleic acid test, antibody testing is also a method to detect COVID-19. Among antibody tests, the most common is the enzyme-linked immunosorbent assay (ELISA). ELISA uses the mechanism of antigens and antibodies binding to detect the presence of antibodies. Before an antigen and an antibody attach, a colored substrate are bind to antigen. After an antigen and an antibody attach, the presence of antibody will have colored result. In the experiment of ELISA COVID-19 detection, the experimental group used covid-19 virus spike protein as a target, and measured the absorbance by adding IgG and substrate, and finally calculated the optical density (OD) value [14]. The ELISA method can help people to determine if they have COVID-19 and whether they are asymptomatic. However, the antibody test can determine if the person is carrying a coronavirus, but it is impossible to determine if the coronavirus is SARS-CoV-2.

\subsubsection{Colloidal Gold Immunochromatographic Assay}

The colloidal gold immunochromatographic assay detect $\mathrm{IgM} / \mathrm{IgG}$ in a rapid, simple, and low-cost way. The experiment results shows that the colloidal gold immunochromatographic assay has a sensitivity of $71.1 \%$ and a specificity of $96.2 \%$ in patients suspected of acquiring COVID-19, and a specificity of $100 \%$ in healthy individuals with 0 positive results [15]. The result indicates that the colloidal gold immunochromatographic assay has high sensitivity and specificity. It can be used before nucleic acid testing to develop the accuracy of the result. Those healthy individuals should have 0 positive results, while nonzero results should undergo nucleic acid testing.

\subsubsection{Two Lateral Flow Immunoassay}

Two Lateral Flow Immunoassay is a quick and easy way to help people detect asymptomatic infections. Two Lateral Flow Immunoassay Tests are important because traditional PCR can only detect people infected with COVID-19 but cannot help identifying asymptomatic people who have been exposed to an infected person. The Two Lateral Flow Immunoassay Test diagnose COVID-19 infected or exposed individuals who have developed antibodies by detecting $\operatorname{IgM}$ and $\operatorname{IgG}$. This method has a sensitivity of $87.7 \%$, which is much higher than that only use of single Lateral Flow Immunoassay Tests, so using Two Lateral Flow Immunoassays can greatly improve the accuracy of the test results $\left[{ }^{16}\right]$. However, no results of shows which two combination of the Lateral Flow Immunoassays are the most efficient. It might be the future research direction.

\subsubsection{Combination of Various Methods to Detect Covid-19}

Nucleus acid test methods are used in conjunction with antibody detection methods to accurately detect COVID- 
19. CRISPR-based DETECTR lateral flow assay is a method that uses CRISPR and lateral flow assay, which has $95 \%$ sensitivity and $100 \%$ specificity. This method can effectively replace RT-PCR and obtain highly accurate results without complex instrumentation [16]. Currently, many countries use the nucleus acid test combined with an antibody test to accurately diagnose COVID-19. Therefore, future detected methods might be a combination of existing methods. The disadvantage of a method can be compensated with the advantage of another method. Combining the strengths and limitations of the two methods could contribute to the development of the most effective method for detecting COVID-19 in the future.

\subsection{Conclusion}

The covid-19 global dissemination has demonstrated the value of understanding the structure and pathogenesis of the covid-19 virus. This paper basically structures COVID-19, the pathogenesis of COVID-19, covid-19 detection methods for several available antiviral drugs. Currently, researchers have a general understanding of the pathogenicity of COVID-19 and find out some assays for COVID-19 detection; however, a rapid, highly sensitive, and specific detection method is still the direction of future research. Many drugs tested for COVID-19 have been found to have a therapeutic effect on COVID-19 but have certain adverse effects. The research on new drugs for COVID-19 treatment is still very urgent. Before there are breakthroughs in COVID19 treatment options, the use of masks and social distance remain the most effective practices to prevent COVID-19 transmission. It could be a long fight against COVID-19, and it will require people around the world to work together in a concerted effort.

\section{Antiviral drugs}

\subsection{Lopinavir}<smiles>Cc1cccc(C)c1OCC(=O)N[C@@H](Cc1ccccc1)[C@H](O)C[C@@H](Cc1ccccc1)NC(=O)[C@@H](C(C)C)N1CCCNC1=O</smiles>

Figure 1. The structural formula of lopinavir.<smiles>CC(C)c1nc(CN(C)C(=O)N[C@H](C(=O)N[C@H](Cc2ccccc2)C[C@@H](O)[C@H](Cc2ccccc2)NC(=O)OCc2cncs2)C(C)C)cs1</smiles>

Figure 2. The structural formula of ritonavir.

The molecular formula of lopinavir (Figure 1) is $\mathrm{C} 37 \mathrm{H} 48 \mathrm{~N} 4 \mathrm{O} 5$, and its molecular weight is 628.80 [17]. The co-formulation of lopinavir and ritonavir (Figure 2), named Kaletra, was approved by FDA to be used in the therapy of HIV infection, which Abbott Laboratories, USA developed. And both lopinavir and ritonavir are inhibitors of HIV-1 protease, an enzyme that cleaves the HIV polyproteins Gag and Gag-Pol by bond hydrolysis.

Lopinavir can lead to the production of immature, non-infectious viral particles [17]. However, it is rapidly degraded by the host proteases in vivo. Therefore, ritonavir, which helps lopinavir remain active for a longer time via inhibiting the metabolizing enzyme cytochrome $\mathrm{P} 450$, is needed to be used together [18]. Although coronavirus has the different main proteases with HIV, it has been found that lopinavir has similar binding energies against the SARS-CoV-2 and HIV-1 proteases [18]. To complement the formation of the viral replication complex, coronaviruses need to cleave the ORF-1 polypeptide, which is cleaved by papain-like protease (PLpro) and 3C-like protease (3CLpro) to produce non-structural proteins like RNA-dependent RNA polymerase (RdRp) [19]. In vitro studies against coronaviruses, lopinavir/ritonavir have shown inhibitory activity on the 3CLpro enzyme [20].

Currently, a lot of countries are included lopinavir/ritonavir in their clinical guidelines for treating COVID-19. And as of 14th June 2020, there are 48 clinical trials have been registered around the world [20]. However, some adverse effects need to be noted. The most common adverse effect is diarrhea and gastrointestinal disturbance. And other symptoms like elevated liver enzymes, dyslipidemia, asthenia, headache, and skin rashes are minor side effects [21].

\subsection{Chloroquine \& Hydroxychloroquine}<smiles>CCN(CC)CCCC(C)Nc1ccnc2cc(Cl)ccc12</smiles>

Figure 3. The structural formula of chloroquine. 
<smiles>CCN(CCO)CCCC(C)Nc1ccnc2cc(Cl)ccc12</smiles>

Figure 4. The structural formula of hydroxychloroquine. Chloroquine (CQ) (Figure 3) has long been used to treat malaria and amebiasis, as well as some inflammatory diseases such as systemic lupus erythematosus (SLE) and rheumatoid arthritis (RA). It was synthesized in 1934 by Hans Andersag at Bayer. First synthesized in 1946, hydroxychloroquine (Figure 4) is a chloroquine derivative made by introducing $\mathrm{OH}$-group into chloroquine.20 Compared to CQ, HCQ has a similar chemical structure, acting as a weak base and immunomodulator, and antiviral activity but with less toxicity.

As weak diprotic bases, CQ and HCQ can concentrate in the endosomes, increasing the $\mathrm{pH}$ of the endosomal fluid. But the viral enzymes responsible for proteolysis and post-translational modification of nascent proteins have the best activity with acidic $\mathrm{pH}$ of the endosomes. Therefore, the increased $\mathrm{pH}$ can affect virus replication or assembly and release. Moreover, a study suggested CQ and HCQ cause the failure of further transport of virions to the ultimate releasing site by blocking the endosome maturation [21,22]. In addition, $\mathrm{CQ}$ and $\mathrm{CHQ}$ can interfere with the glycosylation of ACE2, which is the host receptor for the virus. And the under-glycosylated ACE2 has a poor affinity to SARS$\mathrm{CoV}$ spike protein, preventing the virus from entering the host cell $[19,21,22]$. Autophagy is a lysosomedependent degradative pathway. As weak bases, CQ and HCQ can inhibit lysosomal acidification, leading to blocking the fusion of autophagosomes with lysosomes as well as subsequent autophagic degradation. And the inhibition of autophagy may stimulate the generation of superoxide, accumulation of ubiquitin-conjugated protein, and apoptosis, thus inhibiting virus replication [19]. CQ and HCQ treatment may reduce the release of massive cytokines, such as TNF- $\alpha$ and IL-6, which aggravate the disease by anti-inflammatory and immunomodulatory effects [19].

Although CQ and HCQ are potential treatment to COVID-19 and have been included in the guidelines of many countries like China, there is very few evidence can prove the efficacy of CQ and CHQ. Even some studies showed reverse results [22]. And CQ/HCQ therapy may have serious adverse effects. A study indicated the possibility of adverse effects like severe cardiac side effects, including QT prolongation, Torsade de Pointes, and arrhythmia [23]. And other adverse effects include retinopathy, bleaching of hair, pruritus, headaches, dizziness, and gastrointestinal upset [22]. In view of the uncertainty of the efficacy and the seriousness of the adverse reactions, We should treat this treatment cautiously.

\subsection{Remdesivir}



Figure 5. The structural formula of remdesivir.

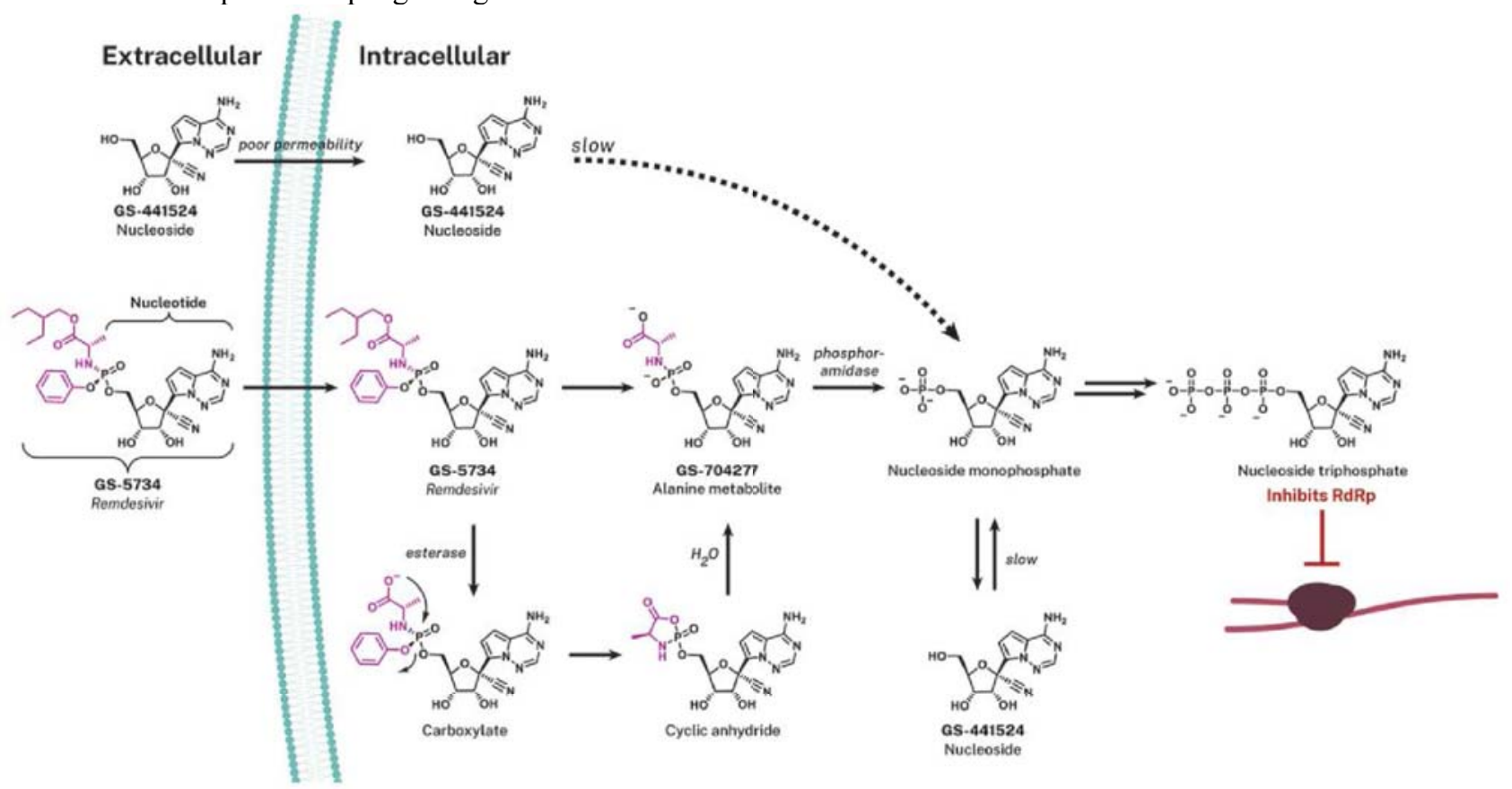

Figure 6. Remdesivir and its intracellular conversion [7]. 
As an adenosine analog, remdesivir (GS-5734) (Figure 5) was developed by Gilead Sciences Inc, originally used to fight the Ebola virus. Apart from the Ebola virus, remdesivir can resist other RNA viruses with its broadspectrum antiviral activity [4]. Remdesivir is metabolized into an alanine metabolite (GS-704277) within cells and then processed into the monophosphate derivative and the active nucleoside triphosphate (NTP) derivative. NTP is an adenosine triphosphate (ATP) analogue, and it can compete with the natural substrate of ATP, which selectively inhibits viral RNA-dependent RNA polymerase (RdRp) (Figure 6). Further, the inhibition is achieved by mistakenly integrating NTP into viral nascent RNA chains by RdRp, leading to the termination of delayed RNA chain during viral replication [24].

A vitro study showed remdesivir could inhibit the replication of SARS-CoV-2, suggesting the potential to treat COVID-19 [25]. What's more, according to the results of the initial Phase 3 clinical trial carried out by Gilead Sciences, remdesivir markedly reduced the mortality rate upon 14 days of treatment [20]. Also, the US Food and Drug Administration (FDA) issued Emergency Use Authorizations for the use of remdesivir against COVID-19 on May 1, 2020 [26]. Although few studies have reported adverse reactions of remdesivir, some clinical trials have shown serious adverse effects such as hepatoxicity, nausea, and acute respiratory failure. Therefore, we need more evidence to verify the effectiveness and security of the drug.

\subsection{Arbidol (Umifenovir)}

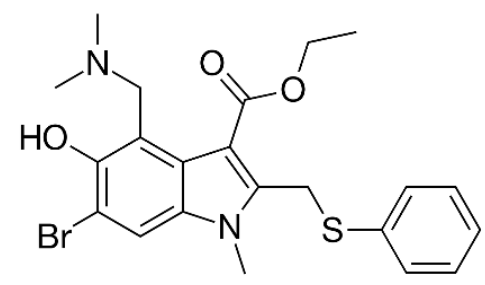

Figure 7. The structural formula of arbidol.

Arbidol (Figure 7), also known as umifenovir, is an indole-derivative. The molecular formula of arbidol is $\mathrm{C} 22 \mathrm{H} 25 \mathrm{BrN} 2 \mathrm{O} 3 \mathrm{~S}$, and the molecular weight is $477.4 \mathrm{~g} / \mathrm{mol}$. Arbidol is a potent broad-spectrum antiviral agent with activity against several enveloped and nonenveloped viruses such as influenza, parainfluenza, coronavirus, polio, and Lassa fever respiratory syncytial virus, etc [27]. It is effective against various pathogenic respiratory viruses with relative safety [18]. Arbidol has been used against influenza and other respiratory viral infections in China and Russia without severe adverse reactions [11]. The mechanism of arbidol against influenza involves binding to hemagglutinin (HA), the major cell-surface glycoprotein, preventing the fusion of the viral membrane, thus blocking virus entry into the cell [18]. Arbidol can blocks viruses from entering cells by interfering with viral binding to host cells and can also inhibit the activity of the virus after entering cells by blocking intracellular vesicle trafficking [27]. Arbidol targets the interaction between viral S-proteins and ACE2 receptors, inhibiting membrane fusion of the viral envelope [21]. A study showed that arbidol binds at the receptor-binding domain $\mathrm{RBD} / \mathrm{ACE} 2$ interface with a high affinity, which induces favorable interactions between them and increases affinity between the RBD and ACE2. Therefore, arbidol binding induces the inflexibility of structure. And the structural rigidity in the viral glycoprotein leads to inhibition of the conformational dynamics required during virus entry [27].

An open-label randomized controlled trial showed more improvement of arbidol with little adverse reaction in peripheral oxygen saturation, requiring ICU admissions, duration of hospitalization, chest CT involvements, WBC, and ESR, compared to Kaletra [27] And another study showed that arbidol might be more effective than lopinavir/ritonavir in treating COVID-19 [26]. Therefore, aebidol has great advantages over other antiviral drugs in treating COVID-19. And now, arbidol has been recommended in the Guideline of preventing and treating COVID-19 in China.

\section{Vaccine}

In today's, people are getting used to the daily new confirmed cases of COVID-19 because it has been classified pandemic for more than 400 days. More than 127 million people are infected globally, and there are thousands of new cases daily. This is still the number one challenge that most countries face, and human beings have fought this disease for years. Thanks to the scientist who dedicates to the research, several vaccines have been invented and are used to ease the chaos.

Before this is written, more than 190 vaccines are being tested in animals and lab experiments in the preclinical stage; and only a few vaccines have been approved for emergency use [28 ]. For COVID-19 particularly, there are three approaches to develop a vaccine [29]. Their distinctions are whether they use the whole virus or bacterium, only the parts of the germ that activate the immune response, or only the genetic material that contains guidance for producing certain proteins rather than the whole virus. And they are classified as the whole-microbe approach, the subunit approach, and the genetic approach.

\subsection{Whole-microbe approaches to develop a vaccine}

For the whole-microbe approach, currently, there are three vaccines classified under this category. The most well-known vaccine is the Inactivated vaccine. The first step in creating a vaccine is to inactivate or destroy the disease-carrying virus or bacterium, or one that is somewhat close to it, using chemicals, fire, or radiation [29]. This technique employs technology that is effective in humans, and this is how flu and polio vaccines are produced, and vaccines can be produced on a fair scale [29]. However, it takes special laboratory equipment to 
safely cultivate the virus or bacterium, can take a very long time to produce, and would almost certainly require two or three doses to be given. Coronavirus is one of the examples of this vaccine and has been widely used in mainland China [30].

A live-attenuated vaccine is classified as a wholemicrobe method. A live-attenuated vaccine employs a living but damaged form of the virus or very similar to it. This form of vaccine includes the measles, mumps, and rubella (MMR) vaccine and the chickenpox and shingles vaccine [29]. This vaccine generates a powerful and long-lasting immune response and develops a better and more robust immune response with a faster onset of immunity than inactivated vaccines [31]. It works by stimulating the body to produce antibodies and memory immune cells in response to the pathogen against which the vaccine protects $[32,33,34,35]$. This method, like the inactivated vaccine, employs similar technologies and can be produced on a large scale. However, such vaccinations may not be appropriate for people with weakened immune systems [29].

A Viral vector vaccine is also a whole-microbe method. This form of vaccine employs a harmless virus to distribute various sub-parts of the germ of interest, known as proteins, to elicit an immune response without causing disease [29]. To do this, the instructions for creating specific sections of the pathogen of interest are incorporated into a healthy virus. The healthy virus then acts as a platform or vector for the protein to enter the body. The antigen triggers the immune response [29]. Examples of this type of vaccine are the OxfordAstraZeneca vaccine, Convidecia, and the Johnson \& Johnson vaccine $[36,37,38]$.

\subsection{Subunit approaches to develop a vaccine}

The subunit approach of developing vaccines is also a crucial method being widely used today. A subunit vaccine is one that only includes the portions of a virus or bacterium that the immune system has to recognize. It does not use a healthy virus as a vector or hold the whole microbe. Often fragments of proteins are injected, the risk of side effects is minimized, but the immune response may also be weaker [39]. Proteins or sugars could be used as subunits. The majority of the vaccines on the pediatric list are subunit vaccines that guard against diseases like whooping cough, tetanus, diphtheria, and meningococcal meningitis. This vaccine is less risky since it is not active [29].

\subsection{Genetic approach to developing a vaccine}

A nucleic acid approach is a novel approach to vaccine development. Before the COVID-19 pandemic, none had gone through the full approvals process for human use, though certain DNA vaccines, including those for specific cancers, were in human trials [29]. Because of the pandemic, a study in this field has moved quickly, and some mRNA vaccines for COVID-19 have received emergency use clearance, which means they can now be given to people outside of clinical trials. Unlike vaccination methods that use damaged or dead whole microbes or portions of them, a nucleic acid vaccine uses only a subset of genetic material that contains the guidance for individual proteins rather than the whole microbe [29]. Human cells use DNA and RNA as guidance to produce proteins. In human cells, DNA is first converted into messenger RNA, which serves as the template for producing various proteins [29]. A nucleic acid vaccine sends a particular series of instructions to our cells, such as DNA or mRNA, instructing them to produce the protein we want our immune system to identify and react to [29].

\subsection{Conclusion}

The vaccination rate is increasing in most countries, but vaccination can be seen as a preventative measure rather than a cure. The most effective way of treating COVID19 is still being researched, and people should not disregard CDC guidance of physical distancing and mask-wearing.

\section{Conclusion}

The global spread of covid-19 has demonstrated the value of understanding the structure and pathogenesis of the covid-19 virus. This paper basically structures COVID-19, the pathogenesis of COVID-19, covid-19 detection methods for several available antiviral drugs. Currently, researchers have a general understanding of the pathogenicity of COVID-19 and find out some assays for COVID-19 detection; however, a rapid, highly sensitive, and specific detection method is still the direction of future research. Many drugs tested for COVID-19 have been found to have a therapeutic effect on COVID-19 but have certain adverse effects. The research on new drugs for COVID-19 treatment is still very urgent. Before there are breakthroughs in COVID19 treatment options, masks and social distance remain the most effective practices to prevent COVID-19 transmission. It could be a long fight against COVID-19, and it will require people around the world to work together in a concerted effort.

\section{References}

1. Oberfeld,B., Achanta, A., Carpenter, K., Chen, P., Gilette,N. M., Langat, P., Said, J. T., Schiff,A. E., Zhou, A. S., Barczak, A. K \& Pillai, S. SnapShot: COVID-19. Cell,181,954-954,(2020).

2. Huang, Y., Yang, C., Xu, X. F., Xu, W, \& Liu, S. W. Structural and functional properties of SARS-CoV2 spike protein: potential antivirus drug development for COVID-19. Acta Pharmacal Sin. 41, (2020).

3. Schoeman, D. \&Fielding, B. C. Coronavirus envelope protein: current knowledge. Virol $J 16$, (2019). 
4. Chen, C. Y. et al. Structure of the SARS coronavirus nucleocapsid protein RNA-binding dimerization domain suggests a mechanism for helical packaging of viral RNA. J Mol Biol 368, 1075-1086, (2007).

5. Nie et al., Multi-organ proteomic landscape of COVID-19 autopsies. Cell, 184, 775-791 e14.(2021).

6. Puntmann et al., Outcomes of Cardiovascular Magnetic Resonance Imaging in Patients recently Recovered From Coronavirus Disease 2019 (COVID-19). JAMA Cardiol, 5, (2020).

7. Feldstein et al., Characteristics and Outcomes of US children and Adolescents with multisystem inflammatory syndrome in Children(MIS-C) Compared with severe acute COVID-19. JAMA, 325, (2021).

8. Frontera et al., Toxic Metabolic Encephalopathy in Hospitalized Patients with COVID-19. Neurocrit Cara.(2021)

9. Corman, V. et al. Detection of 2019 novel coronavirus (2019-nCoV) by real-time RT-PCR. Eurosurveillance 25, (2020).

10. Gorzalski, A. J. et al. High-Throughput Transcription-mediated amplification on the Hologic Panther is a highly sensitive method of detection for SARS-CoV-2. J. Clin. Virol. 129, 104501 (2020).

11. Zhang, W. et al. CRISPR-Based Approaches for Efficient and Accurate Detection of SARS-CoV-2. Lab. Med. (2020).

12. Diagnosis and Treatment Protocol for COVID-19 (Trial Version 7). http://en.nhc.gov.cn/202003/29/c 78469.htm.

13. Rapid, Ultrasensitive, and Quantitative Detection of SARS-CoV-2 Using Antisense Oligonucleotides Directed Electrochemical Biosensor Chip | ACS Nano.

https://pubs.acs.org/doi/10.1021/acsnano.0c06392\#.

14. Adams, E. R. et al. Antibody testing for COVID-19: A report from the National COVID Scientific Advisory Panel. Wellcome Open Res. 5, (2020).

15. Shen, B. et al. Clinical evaluation of a rapid colloidal gold immunochromatography assay for SARS-Cov2 IgM/IgG. Am. J. Transl. Res. 12, 1348-1354 (2020).

16. W, Z. et al. CRISPR-Based Approaches for Efficient and Accurate Detection of SARS-CoV-2. Lab. Med. 52, 116-121 (2021).

17. KALETRA ${ }^{\circledR} \quad$ (lopinavir/ritonavir) capsules, $<$ https:/www.accessdata.fda.gov/drugsatfda_docs/1 abel/2012/021226s035lbl.pdf> (2011).

18. Simonis, A., Theobald, S., Fätkenheuer, G., Rybniker, J. \& Malin, J. A comparative analysis of remdesivir and other repurposed antivirals against SARS-CoV-2. EMBO Molecular Medicine 13, (2021).
19. Uzunova, K., Filipova, E., Pavlova, V. \& Vekov, T. Insights into antiviral mechanisms of remdesivir, lopinavir/ritonavir and chloroquine/hydroxychloroquine affecting the new SARS-CoV-2. Biomedicine \& Pharmacotherapy 131, 110668 (2020).

20. Samudrala, P. et al. Virology, pathogenesis, diagnosis and in-line treatment of COVID-19. European Journal of Pharmacology 883, 173375 (2020).

21. Singh, T. et al. Drug repurposing approach to fight COVID-19. Pharmacological Reports 72, 14791508 (2020).

22. Sun, J. et al. Advances in the use of chloroquine and hydroxychloroquine for the treatment of COVID19. Postgrad Med 132, 604-613 (2020).

23. Eastman, R. et al. Remdesivir: A Review of Its Discovery and Development Leading to Emergency Use Authorization for Treatment of COVID-19. ACS Central Science 6, 672-683 (2020).

24. Singh, A., Singh, A., Singh, R. \& Misra, A. Remdesivir in COVID-19: A critical review of pharmacology, pre-clinical and clinical studies. Diabetes \& Metabolic Syndrome: Clinical Research \& Reviews 14, 641-648 (2020).

25. Frediansyah, A., Nainu, F., Dhama, K., Mudatsir, M. \& Harapan, H. Remdesivir and its antiviral activity against COVID-19: A systematic review. Clinical Epidemiology and Global Health 9, 123-127 (2021).

26. Lam, S., Lombardi, A. \& Ouanounou, A. COVID-19: A review of the proposed pharmacological treatments. European Journal of Pharmacology 886, 173451 (2020).

27. Nojomi, M. et al. Effect of Arbidol (Umifenovir) on COVID-19: a randomized controlled trial. $B M C$ Infectious Diseases 20, (2020).

28. Steckelberg, A., Florit, G., Alcantara, C. \& Johnson, C. (2021). at $<$ https:/www.washingtonpost.com/graphics/2020/h ealth/covid-vaccine-update-coronavirus/ $>$

29. The different types of COVID-19 vaccines. Who.int at https://www.who.int/news-room/featurestories/detail/the-race-for-a-covid-19-vaccineexplained

30. Wu Z, Hu Y, Xu M, Chen Z, Yang W, Jiang Z, Li M, Jin H, Cui G, Chen P, Wang L, Zhao G, Ding Y, Zhao Y, Yin W. Safety, tolerability, and immunogenicity of an inactivated SARS-CoV-2 vaccine (CoronaVac) in healthy adults aged 60 years and older: a randomised, double-blind, placebo-controlled, phase $1 / 2$ clinical trial. Lancet Infect Dis. (2021)

31. Vaccine Type. Live-attenuated vaccines. (2020).at https://www.hhs.gov/immunization/basics/types/ind ex.html (2021). 
32. Gil, C.et al. A DIVA vaccine strain lacking RpoS and the secondary messenger c-di-GMP for protection against salmonellosis in pigs. (2021).

33. Tretyakova, I., Lukashevich, I., Glass, P., Wang, E., Weaver, S. and Pushko, P. Novel vaccine against Venezuelan equine encephalitis combines advantages of DNA immunization and a live attenuated vaccine. (2021).

34. Zou, J., Xie, X., Luo, H., Shan, C., Muruato, A., Weaver, S., Wang, T. and Shi, P. A single-dose plasmid-launched live-attenuated Zika vaccine induces protective immunity. (2021).

35. PLOTKIN, S. A., ORENSTEIN, W. A., \& OFFIT, P. A. Plotkin's vaccines at http://www.clinicalkey.com/dura/browse/bookChap ter/3-s2.0-C20130189143. (2018).

36. Information about the J\&J/Janssen COVID-19 Vaccine. Centers for Disease Control and Prevention (2021).

https://www.cdc.gov/coronavirus/2019-

ncov/vaccines/different-vaccines/janssen.html (2021).

37. Cdc.gov.[online] at: https://www.cdc.gov/vaccines/acip/meetings/downl oads/slides-2021-01/02-COVID-Villafana.pdf (2021).

38. Zimmer, C., Corum, J. and Wee, S., Coronavirus Vaccine Tracker. [online] Nytimes.com. at: https://www.nytimes.com/interactive/2020/science/ coronavirus-vaccine-tracker.html (2021).

39. Gavi.org. What are whole virus vaccines and how could they be used against COVID-19? athttps://www.gavi.org/vaccineswork/what-arewhole-virus-vaccines-and-how-could-they-be-usedagainst-covid-19 (2021). 